$\mathcal{G S}_{\text {https://doi.org/10.3765/sp.11.9 }}^{\text {Semantics \& Pragmatics Volume 11, Article 9, } 2018}$

This is an EARLY ACCESS version of

Lassiter, Daniel. 2018. Complex sentential operators refute unrestricted Simplification of Disjunctive Antecedents. Semantics and Pragmatics 11(9). https://doi.org/10.3765/sp.11.9.

This version will be replaced with the final typeset version in due course. Note that page numbers will change, so cite with caution. 
EARLY ACCESS

\title{
Complex sentential operators refute unrestricted Simplification of Disjunctive Antecedents*
}

\author{
Daniel Lassiter \\ Stanford University
}

\begin{abstract}
There is a longstanding debate about the status of the principle Simplification of Disjunctive Antecedents (SDA), according to which a counterfactual with a syntactically disjunctive antecedent $[(\phi \vee \psi)>\chi]$ entails a conjunction of counterfactuals $[(\phi>\chi) \wedge(\psi>\chi)]$. This principle is highly intuitive for most examples that have been considered, but it has also been claimed to be subject to empirical counter-examples. However, there are promising pragmatic explanations for the currently known counter-examples, which have led several authors to argue in recent work that SDA is unrestrictedly valid after all. This short piece introduces new data involving sentential operators that impose both upper and lower bounds on confidence, frequency, etc., such as likely but not certain, there is an exactly $n \%$ probability, and usually but not always. These examples show clearly that SDA is not valid tout court. While SDA-supporting interpretations do exist and require an explanation, every theory of counterfactuals also requires an explanation of examples that can only be read in a way that does not support SDA.
\end{abstract}

Keywords: counterfactuals, conditionals, disjunction, pragmatics

\section{Simplification of Disjunctive Antecedents: Detractors and defenders}

Lewis's (1973) semantics for counterfactuals does not validate the principle that Nute (1975) labels 'Simplification of Disjunctive Antecedents' (SDA). Using > to represent the counterfactual connective, I will define SDA as the claim that counterfactuals with the form of (1a) entail (1b). ${ }^{1}$

(1) a. $(\phi \vee \psi)>\chi$

b. $(\phi>\chi) \wedge(\psi>\chi)$

Nute (1975) and Fine (1975) object to Lewis' semantics that SDA is intuitively valid in examples like (2) (slightly modified from Nute).

* Many thanks to Josh Dever and three anonymous reviewers for extremely helpful comments that helped me to improve and streamline this squib.

1 Note that the principle is sometimes given a stronger formulation according to which (1a) is equivalent to (1b). I will only be concerned with the weaker version, which is enough to generate the problems I will discuss. 
(2) If we were to have good weather this summer or the sun were to grow cold before the end of summer, we would have a bumper crop.

(3) a. If we were to have good weather this summer, we would have a bumper crop.

b. If the sun were to grow cold before the end of summer, we would have a bumper crop.

Plainly, it is reasonable to infer from (2) the conjunction of the conditionals in (3). Since (3b) is bizarre, the validity of (1) would provide a clear explanation of the bizarreness of (2), which Lewis' theory does not predict in any straightforward way. SDA is much-discussed and has been explicitly endorsed by many authors from the early days of work on the topic to the present: see, for example, Nute 1975, Ellis et al. 1977, Warmbrōd 1981, Fine 2012, Starr 2014, Willer $2017 .^{2}$

Following up on the early discussion noted above, Loewer (1976) and McKay \& van Inwagen (1977) argue that SDA is not valid. McKay \& van Inwagen give the apparent counter-example in (4):

(4) If Spain had fought with the Axis or the Allies it would have fought with the Axis.

While (5a) is harmless, (5b) seems to be a troubling entailment.

a. If Spain had fought with the Axis it would have fought with the Axis.

b. If Spain had fought with the Allies it would have fought with the Axis.

Examples with the form $(\phi \vee \psi)>\phi$, where $\phi$ and $\psi$ are incompatible, are frequently cited as evidence that SDA is not unrestrictedly valid: see, for example, Nute 1980, 1984, Bennett 2003, van Rooij 2006, Santorio 2017.

2 The issues discussed in this paper are not much affected if we choose a restrictor syntax for conditionals (Lewis 1975, Kratzer 1991a), where the antecedent of a conditional restricts a (possibly silent) operator $O p$ and there is no 'counterfactual connective' per se. In that case, we just need to reframe the question to ask whether a sentence with the LF $O p[\phi \vee \psi][\chi]$ entails sentences with LFs $O p[\phi][\chi]$ and $O p[\psi][\chi]$. Whether this inference comes out does not depend on the syntax directly, but on the details of the semantics of $O p$ and how restriction is modeled. For example, in Kratzer's (1991b) semantics, these inferences would not go through for sentences with no overt operator if the silent operator has the force of epistemic must. However, one could easily devise a variant of the restrictor theory in which they do - for example, by requiring an empty ordering source or using a different silent operator. The classical dialectic around the validity of SDA would then apply equally to the choice among these variants of the restrictor theory. Where complex operators are concerned, the predictions are complex and depend on a number of syntactic and semantic choices, such as whether to use a singly- or doubly-modalized interpretation. I will not go through the various options in this space, but will simply note that there are variants of the restrictor theory that validate SDA unrestrictedly, and others that do not. Users should be careful to ensure that their favored theory is able to make sense of the data introduced in section 2. 
However, there is a very promising explanation for this apparent failure of SDA, which has led some authors to argue that SDA is unrestrictedly valid after all (Warmbrōd 1981, Starr 2014, Willer 2017; see also Fine 2012 for a related diagnosis). Suppose that Spain fights with the Allies is not relevantly possible. Suppose also, with Lewis (1973) and many others, that counterfactuals with impossible antecedents are vacuously true. In that case, both counterfactuals in (5) are unobjectionable, since both are trivially true. So, if SDA is valid then (4) should simply imply that Spain fights with the Allies is not relevantly possible — that is, that (6) is false.

(6) Spain might have fought with the Allies.

This prediction appears to be correct. Someone whose knowledge supports (4) is presumably aware of Spain's fascist leanings during the WWII period, which render the possibility of alliance with the Allies against Hitler exceedingly remote. Likewise, someone who had little background knowledge about the war would be justified in inferring from (4) that (7) is true.

(7) Spain would never have fought with the Allies.

Far from being a refutation of SDA, then, McKay \& van Inwagen's (1977) example (4) is beginning to look like surprising confirmation of this principle's validity. Example (4) conveys exactly what we would expect it to convey, if (a) SDA is valid, and (b) counterfactuals with impossible antecedents are trivially true.

In sum, McKay \& van Inwagen's (1977) purported counter-examples to SDA are often cited as showing that the principle is not valid. However, they do not in fact provide an airtight case for this conclusion, and the empirical situation leaves room for theories of counterfactuals that do validate SDA.

At this point, adopting a theory that validates SDA globally seems very attractive. Such a theory would explain why SDA readings are prominent in (2) and so many other examples in a very straightforward way. Theories that do not validate SDA have more work to do. First, they must explain how both SDA-supporting and classical readings arise. Some theories invoke optional covert operators that convert SDAsupporting disjunctive antecedents into ones that receive a classical interpretation that does not support SDA (e.g., Alonso-Ovalle 2009, Ciardelli et al. 2018, Santorio 2017). Others have posited more or less complicated pragmatic derivations of SDA readings from a theory that, like Lewis', does not support it semantically on any interpretation (e.g., Loewer 1976, Nute 1980, Bennett 2003, Franke 2011). In either case, the net result is that sentences like (2) are ambiguous between a non-SDA interpretation that generates only the reasonable inference in (3a), and an SDAsupporting interpretation that also generates the bizarre (3b). For a brief label, I will call theories in this general class "ambiguity theories".

Ambiguity theories have the additional burden of explaining why the crop example (2) and similar sentences are felt to be false. For them, (2) has two readings: 
a false, SDA-supporting interpretation, and a true interpretation that is contextually equivalent to (3a) (against the background knowledge that good weather is more plausible than the sun growing cold). But why would a listener faced with two ways to interpret (2) focus uncharitably on the false SDA-supporting reading with its bizarre entailment (3b), while ignoring the possibility of assigning (2) a sensible interpretation? Ambiguity theories require an additional explanation of why such examples are not naturally interpreted in the way that ambiguity theories also make available. In contrast, theories that validate SDA have an immediate explanation of the oddity of (2): it entails the plainly false (3b).

At this point the theoretical and empirical situation seems to favor a theory that simply validates SDA. Such a theory is strictly less complex than one that posits an ambiguity; it does not need an additional account of why the ambiguity is not resolved in a maximally charitable way in examples like (2); and, contrary to lore, it is not refuted by McKay \& van Inwagen's (1977) example (4).

\section{Complex operators show that SDA is not generally valid}

However, the empirical situation is worse for SDA than it has appeared. The clever pragmatic defense of the principle against McKay \& van Inwagen's (1977) (4) does not extend to examples with certain complex operators in the consequent.

(8) If Spain had fought with the Axis or the Allies it's likely, but not certain, that it would have fought with the Axis.

If SDA were valid, (8) would entail both of the counterfactuals in (9).

(9) a. If Spain had fought with the Axis it's likely, but not certain, that it would have fought with the Axis.

b. If Spain had fought with the Allies it's likely, but not certain, that it would have fought with the Axis.

(8) is sensible, but both of the purported entailments in (9) are trivially false. So, SDA cannot be valid in general.

The problem extends to other sentential operators that impose both an upper and a lower bound on probability, frequency, generality, etc. For example, (10) clearly does not have the unsatisfiable entailments (10a) or (10b). Likewise, SDA would generate bizarre inferences for (11) and (12).

(10) [Jim likes odd numbers, but is indifferent among them. Betting on the roll of a die, he chose 5.]

If Jim had bet on 1 or 3 , there is an exactly $50 \%$ probability that he would have bet on 3 . 
Complex sentential operators refute unrestricted SDA

a. If Jim had bet on 1 there is an exactly $50 \%$ probability that he would have bet on 3 .

b. If Jim had bet on 3 there is an exactly $50 \%$ probability that he would have bet on 3 .

(11) If it were to rain or snow in New Mexico, it would usually — but not always - rain.

a. If it were to rain in New Mexico, it would usually — but not always — rain.

b. If it were to snow in New Mexico, it would usually — but not always — rain.

(12) If a classical musician switched to playing jazz or hip-hop she would normally — but not necessarily — switch to playing jazz.

a. If a classical musician switched to playing jazz she would normally — but not necessarily — switch to playing jazz.

b. If a classical musician switched to playing hip-hop she would normally - but not necessarily — switch to playing jazz.

These observations refute theories on which SDA is unrestrictedly valid. They are consistent with ambiguity theories, where counterfactuals with syntactically disjunctive antecedents are semantically or pragmatically ambiguous between a reading which validates SDA and one which does not.

The same problems apply to the prima facie plausible application of SDA to indicative conditionals. While indicative SDA would correctly characterize the intuitive interpretation of (13), none of (14)-(16) entail the conditionals that indicative SDA would assign them.

(13) If you eat sea cucumber or dung beetles you'll get a stomachache.

(14) [Jim likes odd numbers, but is indifferent among them. We don't know how he bet.]

If Jim bet on 1 or 3 there is an exactly $50 \%$ probability that he bet on 3 .

(15) If it rained or snowed in New Mexico last week, it's likely — but not certain - that it rained.

(16) If a person is driven by starvation to eat either dirt or insects, they usually — but not always - choose to eat insects.

Indicative conditionals may also default to an interpretation that licenses SDA. However, this is apparently due to semantic ambiguity or pragmatic enrichment, as we would expect (for example) from Alternative/Inquisitive Semantics or from an implicature-based account. 
Daniel Lassiter

\section{Conclusion}

The data in Section 2 indicate that every theory of counterfactuals must be able to generate an interpretation for counterfactuals with disjunctive antecedents that does not support SDA and that is able to make sense of upper- and lower-bounding operators. This result provides compelling support for ambiguity theories over theories that validate SDA tout court.

One puzzle for ambiguity theories mentioned in Section 1 remains, though. Why, when presented with a sentence like $(2) /(17)$ that has a sensible interpretation contextually equivalent to (18a) and a bizarre interpretation (18b), would an interpreter fixate on the bizarre interpretation?

(17) If we were to have good weather this summer or the sun were to grow cold before the end of summer, we would have a bumper crop.

(18) a. good weather $>$ bumper crop

b. $\quad($ good weather $>$ bumper crop $) \wedge($ sun grows cold $>$ bumper crop $)$

As Loewer (1976), Nute (1980) suggest, there must also be something pragmatically defective about the use of sentence (17) to convey the sensible interpretation (18a). Ambiguity theories owe a detailed explanation of what precisely is wrong.

My suspicion is that the explanation is related to Grice's (1989) Maxim of Manner. If the speaker intended to communicate the meaning in (18a), why (to paraphrase Grice) would she choose the rigamarole in (17) when there is a simpler, unambiguous expression available - (19) — that conveys this content without the pointless detour about the sun's growing cold?

(19) If we were to have good weather this summer, we would have a bumper crop. If this style of explanation works out, we can explain the bizarreness of (17) by appealing to independently motivated reasons why both of its interpretations would be infelicitous: (18a) is ruled out by Manner, and (18b) by Quality. However, more work will be needed to defend such a pragmatic account in detail and to confront it with a wider range of examples.

\section{References}

Alonso-Ovalle, Luis. 2009. Counterfactuals, correlatives, and disjunction. Linguistics and Philosophy 32(2). 207-244. https://doi.org/10.1007/s10988-009-9059-0.

Bennett, Jonathan Francis. 2003. A philosophical guide to conditionals. Clarendon Press Oxford.

Ciardelli, Ivano, Linmin Zhang \& Lucas Champollion. 2018. Two switches in the theory of counterfactuals: A study of truth conditionality and minimal change. To appear in Linguistics and Philosophy. 
Complex sentential operators refute unrestricted SDA

Ellis, Brian, Frank Jackson \& Robert Pargetter. 1977. An objection to possibleworld semantics for counterfactual logics. Journal of Philosophical Logic 6(1). 355-357. https://doi.org/10.1007/BF00262069.

Fine, Kit. 1975. Critical Notice: Counterfactuals. Mind 84(1). 451-458. https: //doi.org/10.1093/mind/LXXXIV.1.451.

Fine, Kit. 2012. Counterfactuals without possible worlds. Journal of Philosophy 109(3). 221-246. https://doi.org/10.5840/jphil201210938.

Franke, Michael. 2011. Quantity implicatures, exhaustive interpretation, and rational conversation. Semantics and Pragmatics 4. 1-82. https://doi.org/10.3765/sp.4.1.

Grice, H. Paul. 1989. Studies in the Way of Words. Harvard University Press.

Kratzer, Angelika. 1991a. Conditionals. In A. von Stechow \& D. Wunderlich (eds.), Semantik: Ein internationales Handbuch der zeitgenössischen Forschung, 651-656. Walter de Gruyter.

Kratzer, Angelika. 1991b. Modality. In Arnim von Stechow \& Dieter Wunderlich (eds.), Semantik: Ein internationales Handbuch der zeitgenössischen Forschung, 639-650. Walter de Gruyter.

Lewis, David. 1973. Counterfactuals. Harvard University Press.

Lewis, David. 1975. Adverbs of quantification. In Edward L. Keenan (ed.), Formal semantics of natural language, 178-188. Cambridge University Press.

Loewer, Barry. 1976. Counterfactuals with disjunctive antecedents. The Journal of Philosophy 73(16). 531-537. https://doi.org/10.2307/2025717.

McKay, Thomas \& Peter van Inwagen. 1977. Counterfactuals with disjunctive antecedents. Philosophical studies 31(5). 353-356. https://doi.org/10.1007/ BF01873862.

Nute, Donald. 1975. Counterfactuals and the similarity of worlds. Journal of Philosophy 72(21). 773-778. https://doi.org/10.2307/2025340.

Nute, Donald. 1980. Conversational scorekeeping and conditionals. Journal of Philosophical Logic 9(2). 153-166. https://doi.org/10.1007/BF00247746.

Nute, Donald. 1984. Conditional logic. In D. Gabbay \& F. Guenther (eds.), Handbook of Philosophical Logic, 387-439. Springer.

van Rooij, Robert. 2006. Free choice counterfactual donkeys. Journal of Semantics 23(4). 383-402. https://doi.org/10.1093/jos/ffl004.

Santorio, Paolo. 2017. Alternatives and truthmakers in conditional semantics. To appear in Journal of Philosophy.

Starr, William B. 2014. A uniform theory of conditionals. Journal of Philosophical Logic 43(6). 1019-1064. https://doi.org/10.1007/s10992-013-9300-8.

Warmbrōd, Ken. 1981. Counterfactuals and substitution of equivalent antecedents. Journal of Philosophical Logic 10(2). 267-289. https://doi.org/10.1007/ BF00248853. 


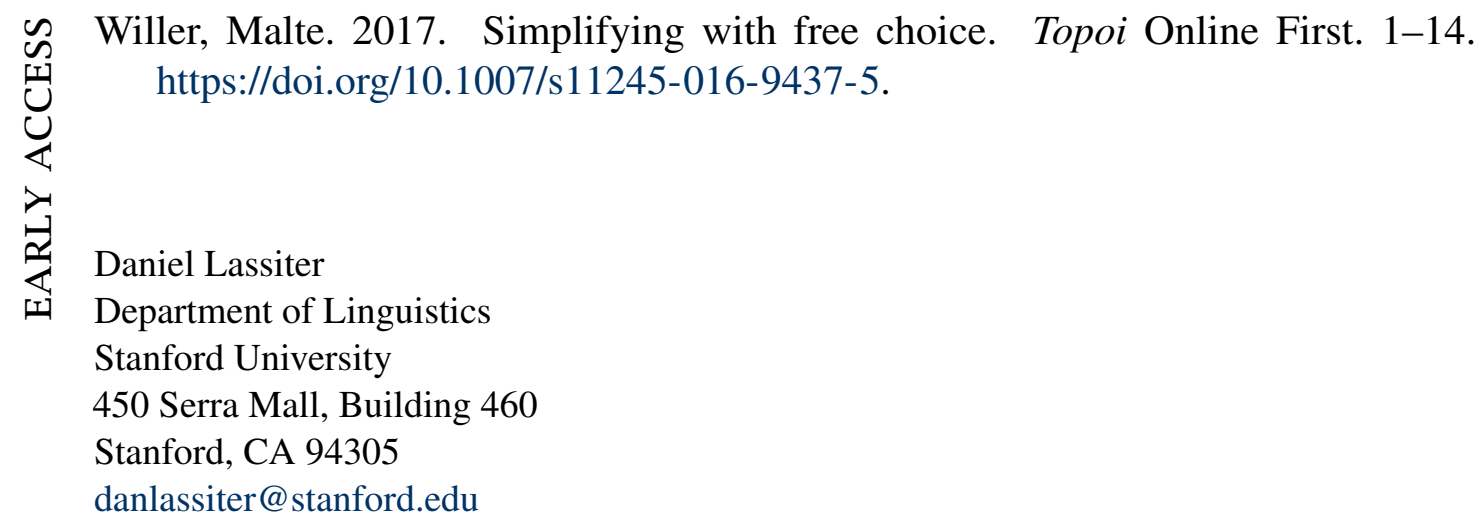

